\title{
Characterization of the Papain-Like Protease p29 of the Hypovirus CHV1-CN280 in Its Natural Host Fungus Cryphonectria parasitica and Nonhost Fungus Magnaporthe oryzae
}

\author{
Qin Xiong, ${ }^{1,2}$ Linqiao Zhang, ${ }^{2,3}$ Justin Waletich, ${ }^{4}$ Linlin Zhang, ${ }^{1}$ Chen Zhang, ${ }^{1}$ Xinyue Zheng, ${ }^{1}$ Yulin Qian, ${ }^{1}$ Zhengguang \\ Zhang, ${ }^{2}$ Yuanchao Wang, ${ }^{2}$ and Qiang Cheng $5, \dagger$ \\ ${ }^{1}$ Co-Innovation Center for Sustainable Forestry in Southern China, College of Biology and the Environment, Nanjing Forestry University, \\ Nanjing 210037, China; ${ }^{2}$ Department of Plant Pathology, Nanjing Agricultural University, Nanjing 210095, China; ${ }^{3}$ Institute of Agricultural \\ Sciences for Lixiahe Region in Jiangsu, Yangzhou 225009, China; ${ }^{4}$ Department of Botany and Plant Pathology, Oregon State University, \\ Corvallis, OR 97331, U.S.A.; and 5The Southern Modern Forestry Collaborative Innovation Center, Nanjing Forestry University, Nanjing \\ 210037, China
}

Accepted for publication 20 December 2018

\begin{abstract}
Cryphonectria hypovirus 1 strain CN280 (CHV1-CN280) was isolated from North China and exhibited typical hypovirulence-associated traits. We previously reported that CHV1-CN280 was more aggressive and had a higher horizontal transmission ability between Cryphonectria parasitica isolates belonging to different vegetative compatibility groups than two other CHV1 hypoviruses (namely, CHV1-EP713 and CHV1-Euro7), thus displaying greater potential for biological control of chestnut blight. The genome sequence of CHV1-CN280 shared approximately $70 \%$ identity with three other hypoviruses (CHV1-EP713, CHV1-Euro7, and CHV1EP721). The coding region for p29, a papain-like protease encoded by CHV1-CN280 hypovirus, displayed an average of only approximately $60 \%$ amino acid identity among them, while the identity between the other three CHV1 isolates was higher than $89 \%$. Protease p29 acted as a virus-encoded determinant responsible for altering fungal host pheno-

sporulation, pigmentation, extracellular laccase activities, and pathogenicity, which is consistent with previous investigations. Subsequently, the potential of CHV1-CN280 p29 as a viral determinant responsible for suppression of host phenotypes in other phytopathogenic fungi such as Magnaporthe oryzae, the causal agent of rice blast disease, was discussed. However, heterologous expression of p29 in M. oryzae induced the opposite effect on sporulation, extracellular laccase activities, and pathogenicity; had no significant effect on pigmentation and mycelial growth; and contributed to extracellular peroxidase activities, suggesting that CHV1-CN280 p29 may disturb a unique regulatory pathway in $C$. parasitica, rather than a basic regulatory pathway conserved in diverse range of fungi. Alternatively, CHV1CN280 p29-mediated modulation of fungal phenotypes may be facilitated by the specific interaction between p29 and a special fungal-host component, which exists only with $C$. parasitica but not M. oryzae.
\end{abstract} types in other CHV1 isolates. In this study, the impacts of CHV1-CN280 p29 expression in virus-free $C$. parasitica were investigated. CHV1CN280 p29 expression in $C$. parasitica resulted in significantly reduced
Keywords: Cryphonectria hypovirus 1, Cryphonectria parasitica, hypovirulence, Magnaporthe oryzae, papain-like protease p29.
The filamentous ascomycete fungus Cryphonectria parasitica, the causative agent of chestnut blight and one of the most ecologically destructive pathogens of chestnut (Castanea spp.), was introduced from eastern Asia to North America and Europe within a 50-year period during the 20th century (Anagnostakis 1982). Fortunately, the spontaneous appearance of hypoviruses with positive-sense, single-stranded RNA genomes showed promise as biological control agents of chestnut blight in European forests and orchards. In addition to reducing virulence

†Corresponding author: Q. Cheng; E-mail: chengqiang@njfu.edu.cn

Funding: This work was supported, in part, by grants provided to Q. Xiong and Q. Cheng by the National Natural Science Foundation of China (numbers 31600512 and 31570639, respectively); to Q. Xiong by the Natural Science Foundation of Jiangsu Province (BK20160923); to C. Zhang, Y. Zhu, and X. Zheng by Students Practice Innovation and Training Program of Nanjing Forestry University (numbers 201710298045Z, 201710298064Z, and 2016NFUSPITP206, respectively); and by the Priority Academic Program Development of Jiangsu Higher Education Institutions

Q. Xiong and L. Zhang contributed equally to this work.

*The $\boldsymbol{e}$-Xtra logo stands for "electronic extra" and indicates that one supplementary table and two supplementary figures are published online.

The author(s) declare no conflict of interest.

(C) 2019 The American Phytopathological Society (hypovirulence), hypovirus infection of $C$. parasitica can cause a stable set of infection-related phenotypic changes, including reduced pigment production, suppressed sexual and asexual reproduction, altered colony morphology, loss of female fertility, and modulated transcription levels of specific host genes, though cytopathic effect or cell death is not observed (Anagnostakis 1982; MacDonald and Fulbright 1991; Nuss 2005). Four distinct Hypovirus species are included in the single genus Hypovirus of the family Hypoviridae. Until now, the complete sequences of four strains belonging to the species Cryphonectria hypovirus 1 (CHV1)-CHV1-EP713 (Shapira et al. 1991), CHV1-Euro7 (Chen and Nuss 1999), CHV1-EP721 (Lin et al. 2007), and CHV1-CN280 (Du et al. 2017) - have been determined. Only one strain each from the other three species (CHV2-NB58, CHV3-GH2, and CHV4SR2) has been completely sequenced. The completion of the wholegenome sequences, along with the feasibility of transforming virusfree strains with a full-length infectious cDNA clone, has facilitated the mapping of hypovirus-encoded symptom determinants and identification of modulators of hypovirus replication from four members of the virus family Hypoviridae.

The prototypic hypovirus CHV1 contains a positive-sense, single-stranded RNA genome of 9.1 to $12.7 \mathrm{~kb}$ that possesses two contiguous open reading frames (ORFs), designated as ORF A and ORF B (Suzuki et al. 2018). ORF A encodes a 69-kDa polyprotein p69, which can be cleaved into p29 and p40 at Gly 248 and Gly 249 by the papain-like cysteine domain of p29. ORF B encodes a larger 
polyprotein that can be cleaved to a $48-\mathrm{kDa}$ papain-like protease p48 with a cysteine protease. In addition to p48 at the $\mathrm{N}$ terminus, ORF B contains an RNA-dependent RNA polymerase and a helicase at the $\mathrm{C}$ terminus ( $\mathrm{Du}$ et al. 2017). The papain-like protease, $\mathrm{p} 29$, excluding the first 24 codons, was previously reported to significantly suppress orange pigmentation, fungal sporulation, and laccase accumulation in the hypovirulent $C$. parasitica strain EP713 but was not necessary for viral replication and the level of hypovirulence (Craven et al. 1993). The first 24 codons of the CHV1 RNA 5'-proximal coding domain were conserved among three fully sequenced genomes of subtype I CHV1 isolates (CHV1EP713, CHV1-Euro7, and CHV1-EP721). Suzuki et al. (2000) found that the deletion of either the first 12 or 24 codons of ORF A within the context of infectious cDNA caused detrimental effects on virus viability and assumed that the first codons of p29 contributed to either translation or RNA synthesis. Chiba et al. (2018) revealed that sequences from the $5^{\prime}$ untranslated regions of hypoviruses (CHV1-EP713, CHV2-NB58, and CHV3-GH2) in C. parasitica have internal ribosomal entry site (IRES) activities based on a dualluciferase assay system, and the CHV1 5'-terminal coding region (first 24 codons) of p29 also acts as a part of the IRES. Suzuki et al. (1999) mapped the p29 symptom determinant domain to a region extending from $\mathrm{Ph}^{25}$ to $\mathrm{Gln}^{73}$, including four strictly conserved cysteine residues: $\mathrm{Cys}^{38}, \mathrm{Cys}^{48}, \mathrm{Cys}^{70}$, and $\mathrm{Cys}^{72}$. Mutation of $\mathrm{p} 29$ residues $\mathrm{Cys}^{70}$ or $\mathrm{Cys}^{72}$, conserved in hypovirus p29, resulted in the loss of both p29-mediated suppressive activity in virus-free transgenic $C$. parasitica and in trans enhancement of RNA accumulation and transmission, suggesting a link between these functional activities. Substitution of a glycine residue for either $\mathrm{Cys}^{38}$ or $\mathrm{Cys}^{48}$ resulted in no qualitative or quantitative changes in virus-mediated symptoms (Suzuki et al. 2003). CHV1-CN280, a North China isolate of CHV1, was recently reported to have a higher horizontal transmission ability between $C$. parasitica isolates belonging to different vegetative compatibility (VC) groups in comparison with other CHV1 isolates, and the genome of CHV1CN280 was sequenced as well as analyzed with other CHV1 isolates by Du et al. (2017).

Surprisingly, the p29 coding region of CHV1-CN280 had a low level of similarity to other reported CHV1 isolates. The amino acid sequence alignment of p29 showed that the identity value between CHV1-280 and other sequenced CHV1 isolates was approximately $60 \%$, whereas the identity value among the other three isolates was more than $89 \%$. CHV1-CN280 p29 differed from most previously characterized p29 in that the length of amino acids is 247 amino acids rather than 248, as well as $\mathrm{Cys}^{38}$ being replaced by an Arg residue in the p29 protein of CHV1-CN280. Moreover, the Nterminal portion (codons 1 to 24) of CHV1-CN280 p29 had significant differences from those of the other isolates, with only $\mathrm{Met}^{1}, \mathrm{Leu}^{13}, \mathrm{Asp}^{23}$, and $\mathrm{Pro}^{24}$ being identical. However, codons 2 to 24 of the p29 protein of CHV1-EP713 were important for IRES activities (Chiba et al. 2018). Cys ${ }^{162}$ and His ${ }^{215}$, the putative protease active site residues of $\mathrm{p} 29$, along with $\mathrm{Cys}^{48}$, Cys ${ }^{70}$, and $\mathrm{Cys}^{72}$, which have been shown to be critical for the function of $\mathrm{p} 29$, are conserved. It was of interest to test whether CHV1-CN280 p29, sharing low similarities with the other three CHV1 isolates, would also influence a range of host functions (e.g., to determine whether CHV1-CN280 p29 is also responsible for pigmentation, asexual sporulation, laccase accumulation, and hypovirulence).

To date, disease management strategies have mainly relied on fungicide usage and resistant plant cultivar deployment. Given that the application of such chemicals is becoming increasingly problematic due to the emergence of fungicide-resistant strains and the drug-induced toxicological risks to public health, and that resistant cultivars become susceptible to these pathogenic diseases within a few years of release (Fisher et al. 2018; Rimbaud et al. 2018), it is necessary to develop alternative methods such as mycovirus-related biocontrol against these diseases (Xie and Jiang 2014). The approach of transfecting protoplasts of different virus-free fungal host species with hypovirus cDNA or hypovirusencoded symptom determinant domains holds promise for the expanded utility of virus-mediated hypovirulence for controlling fungal pathogenicity (Chen et al. 1996). For example, Magnaporthe oryzae chrysovirus 1 strain A (MoCV1-A) is the causal agent of growth repression and hypovirulence of the rice blast fungus Magnaporthe oryzae. The heterologous expression of MoCV1-A ORF4 protein in human-pathogenic Cryptococcus neoformans and the model eukaryote Saccharomyces cerevisiae both resulted in fungal growth defects, suggesting the potential of the ORF 4 product as a new pharmaceutical protein (Urayama et al. 2012, 2014). Xie et al. (2016) reported that infection with the victorivirus Helminthosporium victoriae virus $190 \mathrm{~S}$ (HvV190S) induced a hypovirulent phenotype in a heterologous plant pathogenic host, Cryphonectria parasitica, suggesting it might be possible to convert other economically important plant-pathogenic fungi to a state of hypovirulence using HvV190S. An infectious cDNA clone of CHV1-EP713 hypovirus was successfully transformed into two additional genera, Valsa ceratosperma and Phomopsis G-type, within the same order, Diaporthales, as $C$. parasitica, by biolistic bombardment. The generated transformants exhibited altered colony morphology and, more importantly, reduced virulence, suggesting a potential for hypoviruses as biological control agents in plant-infecting fungal pathogens other than the natural fungal host (Sasaki et al. 2002).

Considering that CHV1-CN280 p29 is assumed to be critical for the hypovirulence symptoms, the second objective of this study was to obtain further insight into CHV1-CN280 p29 as a viral determinant responsible for modulation of host functions in other phytopathogenic fungi. The filamentous fungus $M$. oryzae is the causal agent of rice blast disease, the most serious and devastating disease of rice worldwide (Dean et al. 2012). M. oryzae is an extremely important "model fungi" for studying fungus-plant interactions. Unfortunately, methods for artificial virus introduction into $M$. oryzae have not yet been developed (Eusebio-Cope et al. 2015). Moreover, the hypovirus-encoded protein p29 was found to have a differential impact on phenotypic types in different fungal species closely related to $C$. parasitica within the family Cryphonectriaceae, including two pathogens of eucalyptus, C. cubensis and C. havanensis; a pathogen of oak, Endothia gyrosa; and the nonpathogenic $C$. radicalis (Chen et al. 1996). However, the species $M$. oryzae shares the same order, Diaporthales, with $C$. parasitica but belongs to a different family. In this study, $M$. oryzae was chosen to further evaluate whether CHV1$\mathrm{CN} 280$ p29 could exhibit broad-spectrum virus-mediated hypovirulence in a nonnatural host.

\section{MATERIALS AND METHODS}

Fungal strains and growth conditions. The $C$. parasitica strain CHV1-CN280 was a North China isolate of CHV1. The virusfree virulent $C$. parasitica strain EP155 (ATCC 38755) was used as the wild type for transformation in this study. All $C$. parasitica strains, including transformants, were maintained on potato dextrose agar (PDA) plates under ambient light at $25^{\circ} \mathrm{C}$ (Gao et al. 2013). Mycelia were collected for the purification of doublestranded (ds)RNA and the extractions of genomic DNA and total RNA, after growing in potato dextrose broth (PDB) for 4 days under similar conditions. Mycelia used for spheroplast preparation were harvested after inoculating $C$. parasitica PDA plugs into a 500-ml flask containing $200 \mathrm{ml}$ of fresh EP complete liquid medium at $\mathrm{pH}$ 5.6 (Puhalla and Anagnostakis 1971) and cultured without shaking for another 3 days at room temperature. For the mycelial growth assay, 3-by-3-mm hyphal tip plugs of the CHV1-CN280 p29expressing transformants, in parallel with those of untransformed wild-type EP155 and hypovirulent strain CHV1-CN280, were transferred from 3-day-old PDA plates and inoculated on fresh PDA plates $\left(7.5 \mathrm{~cm}\right.$ in diameter), followed by incubation at $25^{\circ} \mathrm{C}$ in 
the dark. The colony radial growth was measured and photographed after 7 days of incubation. Similarly, for the conidiation assay, conidia were prepared by flooding 14-day-old PDA Petri dish cultures with $2 \mathrm{ml}$ of distilled water and softly scraping the colony surface with a spatula. Spore suspensions were then filtered through four-layer-thick lens paper and counted using a hemocytometer. For the laccase activity assay, the same mycelial plugs were maintained on PDA medium supplemented with tannic acid as a substrate at $25^{\circ} \mathrm{C}$ for 4 days in the dark (Rigling et al. 1989).

M. oryzae wild-type strain Guy11 and CHV1-CN280 p29expressing transformants were cultured on complete medium (CM) (10 $\mathrm{g}$ of D-glucose, $2 \mathrm{~g}$ of peptone, $1 \mathrm{~g}$ of yeast extract, $1 \mathrm{~g}$ of casamino acids, $50 \mathrm{ml}$ of $20 \times$ nitrate salts, $1 \mathrm{ml}$ of trace elements, $1 \mathrm{ml}$ of vitamin solution, $15 \mathrm{~g}$ of agar, and distilled water added to 1 liter) (Talbot et al. 1993) for 6 days at $28^{\circ} \mathrm{C}$ to assess growth and colony characteristics. PDA, $10 \%$ (vol/vol) V8 medium (V8) ( $100 \mathrm{ml}$ of $\mathrm{V} 8$ juice and $1 \mathrm{~g}$ of $\mathrm{CaCO}_{3}$, centrifuged at 2,500 rpm for 5 min to obtain clear supernatants and brought to 1 liter in volume, and $15 \mathrm{~g}$ of agar), and SDC ( $100 \mathrm{~g}$ of straw decoction into 1 liter of double-distilled $\mathrm{H}_{2} \mathrm{O}, 40 \mathrm{~g}$ of corn meal, and $15 \mathrm{~g}$ of agar) were also used (Qi et al. 2012). Liquid CM was used to harvest the mycelia for genomic DNA and total RNA extractions. To investigate the radial colony growth, mycelial plugs ( 3 by $3 \mathrm{~mm}$ ) of the CHV1-CN280 p29-expressing transformants and wild type were transferred from 6-day-old CM plates and inoculated on fresh media (CM, PDA, SDC, and V8) followed by incubation at $28^{\circ} \mathrm{C}$ with a $12-\mathrm{h}$ interval photophase. The radial growth was measured after incubation for 6 days. To promote conidiation, strains were cultured on SDC medium for 1 week in the dark, followed by 3 days of continuous illumination under fluorescent light. Laccase activity was moni-

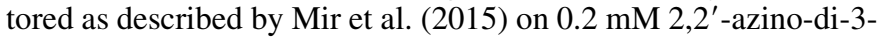
ethylbenzathiazoline-6-sulfonate (ABTS) (A1888; Sigma) agar plates with or without $0.5 \mathrm{mM}$ copper sulfate using mycelial blocks ( 3 by $3 \mathrm{~mm}$ ) at 3 days postinoculation. Mycelial blocks were placed on CM medium containing 200-ppm Congo Red (CR) (860956; Aldrich) agar plates for 5 days to measure peroxidase secretion. Peroxidase and laccase activities were measured by combining $1 \mathrm{ml}$ of reaction mixture $(0.05 \mathrm{M}$ acetate buffer [pH 5.0] and $20 \mathrm{mM}$ ABTS) with 3-day-old CM liquid culture filtrate $(200 \mu \mathrm{l})$, followed by incubation for $5 \mathrm{~min}$ at $25^{\circ} \mathrm{C}$. Absorbance was evaluated at a $420-$ nm wavelength using a spectrophotometer (Mir et al. 2015).

All experiments in this section were performed in three independent biological experiments with three replicates in each test.

Purification of dsRNA and construction of CHV1CN280 p29-expressing vector. The mycelia harvested from PDB were manually separated from culture media with forceps, and excess moisture was removed by pressing the mycelia between paper towels. The following steps for the purification of dsRNA of hypovirus CHV1-CN280 isolate were followed using the protocol described by Castillo et al. (2011). Viral dsRNA molecules were eluted by putting an agarose piece in an Eppendorf tube with tripledistilled water and incubating at $4^{\circ} \mathrm{C}$ overnight. The agarose-free dsRNA molecules were concentrated by ethanol precipitation. cDNA clones were generated using a RevertAid first-strand cDNA synthesis kit (Thermo Scientific) according to the manufacturer's protocol. For cDNA polymerase chain reaction (PCR) amplification, we used Go Taq DNA polymerase with colorless buffer (Promega). Then, the cDNA fragment of full-length CHV1-CN280 was cloned into the pMD19-T vector (TaKaRa).

A 741-bp band containing the full-length CHV1-CN280 p29 gene was amplified by PCR with Primer STAR Pfu Taq DNA polymerase (TaKaRa), using the above plasmid pMD19-T::CHV1CN280 cDNA as a template, with primer pair Cpp29-KpnI-F/ Cpp29-SacI-R, which incorporated restriction sites for KpnI and SacI, respectively. The purified PCR fragment was double digested with KpnI and SacI, then fused with a KpnI- or SacI-digested destination vector pCPXHY1, which constitutively drove gene expression with the $C$. parasitica glyceraldehyde-3-phosphate dehydrogenase promoter and carried the hygromycin (Hyg) resistance gene. The final expression construct, pCPXHY1:: CHV1-CN280-p29, was generated and then used to transform the protoplasts of $C$. parasitica wild-type strain EP155, as described by Churchill et al. (1990). Similarly, the expression construct pCB1532::CHV1-CN280-p29, conferring sulfonylurea resistance, was generated with the primer pair Mop29-HindIII-F/Mop29$K p n I-\mathrm{R}$ and used for protoplast-mediated transformation of M. oryzae Guy11 following a previously described protocol (Talbot et al. 1993).

Screening of the putative transformants. After transformation, the colonies growing on selective regeneration medium were considered as primary putative CHV1-CN280 p29-expressing transformants. All primary colonies were transferred onto PDA plates containing either Hyg B (Roche Diagnostics) at $150 \mu \mathrm{g} / \mathrm{ml}$ or sulfonylurea at $100 \mu \mathrm{g} / \mathrm{ml}$ to screen stable transformants. Genomic DNA was extracted from mycelia of the putative stable transformants and nontransformed controls using Invitrogen genomic DNA extraction kits, which were used as PCR amplification templates. Characterization of transformants by PCR was performed with the CHV1-CN280 p29-specific primer (p29-F/p29-R) to detect the integration of the $p 29$ gene.

Southern blotting analysis. For Southern blotting analysis, genomic DNA extracted from $C$. parasitica wild-type strain EP155, $M$. oryzae wild-type strain Guy11, and the CHV1-CN280 p29expressing transformants were digested with EcoRI, EcoRV, and HindIII, separated by electrophoresis on $0.8 \%$ agarose gel overnight, then transferred onto a positively charged nylon transfer Hybond TM-N ${ }^{+}$membrane (Amersham, Biosciences UK Limited). Only one recognition site of each of three restriction enzymes (EcoRI, EcoRV, and HindIII) was present within the two transforming plasmids, and no recognition sites in the p29 fragment. The labeled probe was amplified from cDNA of full-length CHV1$\mathrm{CN} 280$ by primer pair $\mathrm{p} 29-\mathrm{F} / \mathrm{p} 29-\mathrm{R}$ (Supplementary Table $\mathrm{S} 1$ ). The hybridization and detection were performed with the digoxigenin high prime DNA labeling and detection Starter Kit II (Roche Applied Science) according to the manufacturer's instructions.

Semiquantitative reverse-transcription PCR and quantitative real-time $P C R$ analyses. Total RNA samples were isolated using NucleoSpin RNAII (Macherey-Nagel). In total, $5 \mathrm{mg}$ of total RNA was treated with DNase I (TaKaRa) prior to cDNA synthesis to avoid DNA contamination. First-strand cDNA was synthesized from the treated RNA using the synthesis system of M-MLV reverse transcription (Invitrogen) and oligo(dT) 15 primers (TaKaRa). CHV1-CN280 p29 was amplified with primers RTp29-F and RT-p29-L for a reverse-transcription (RT)-PCR assay. As the endogenous controls, the 18S ribosomal RNA (rRNA) and actin genes were amplified with primers RT-Cp18S-F/R and RT-MoActin-F/R, respectively. Quantitative real-time (qRT)-PCR was performed with qRT-PCR primers using the ABI 7500 Fast Real-Time PCR System (Applied Biosystems) following the manufacturer's instructions. $\alpha$-Tubulin was used as an internal standard for $C$. parasitica and actin was used for M. oryzae.

To evaluate the effect of CHV1-CN280 p29 overexpression on the relative abundance of gene transcripts, total RNA of wild-type Guy11, EP155, and CHV1-CN280 p29-expressing transformants was extracted as shown above. The expression level of three genes (lac1, lac2, and lac3) closely associated with laccase activity in $C$. parasitica and two genes (GenBank accession numbers $M G G_{-} 11608$ and $M G G_{-} 13464$ ) in $M$. oryzae were selected for further analysis. Moreover, the five genes (GenBank accession numbers $M G G \_07790, M G G \_13291, M G G \_11856, M G G \_08200$, and $M G G \_01924$ ) of $M$. oryzae in connection with extracellular peroxidase activities were selected. The experiment was carried out three times, and each qRT-PCR had three replicates.

Plant infection assays. Pathogenicity assessment of $C$. parasitica was performed in the laboratory according to the procedure 
described by Kim et al. (1995), with minor modifications. The dormant Chinese chestnut stems (2 to $3 \mathrm{~cm}$ in diameter) were collected from 10 healthy trees (located in Nanjing Botanical Garden in China), cut to approximately $50 \mathrm{~cm}$ length, and surface sterilized with $70 \%$ ethanol. A small hole (approximately $8 \mathrm{~mm}$ ) per stem was made by removing a plug of bark to the depth of the cambium with a hole punch $(9 \mathrm{~mm}$ in diameter). Mycelial plugs, derived from actively growing margins of cultures of either $C$. parasitica wild-type EP155, hypovirus CHV1-CN280, or CHV1CN280 p29-expressing transformants, were randomly inserted into the wounded holes. The inoculated stems were then covered with soaked cotton wool, sealed with adhesive tape to prevent desiccation, and kept in a sterilized container at room temperature (20 to $22^{\circ} \mathrm{C}$ ) with high humidity (approximately 95\%) in the dark. Simultaneously, the agar block was used as a control. Each strain was inoculated into three stems. The major and minor semiaxes of the developing cankers were measured and photographed after 5 weeks of incubation. In addition, the canker area was calculated using the formula for an ellipse $(S=1 / 4 \pi \mathrm{LD}$, where $\mathrm{L}$ and $\mathrm{D}$ represented the major and minor semiaxis, respectively).

For the pathogenicity assessment of $M$. oryzae, 15-day-old susceptible rice (Oryzae sativa 'CO39') and 7-day-old barley (Hordeum vulgare 'Four-arris') seedlings were prepared for plant infection assays. Conidia used for pathogenicity assays were prepared as shown below. The concentration of conidial suspensions was adjusted to $1 \times 10^{5}$ spores $/ \mathrm{ml}$ and was supplemented with $0.2 \%(\mathrm{wt} / \mathrm{vol})$ gelatin. In total, $5 \mathrm{ml}$ was sprayed on the intact rice leaf sheath with the help of the larynx-like sprayer tool, and inoculated rice leaves were placed in a moist chamber at $28^{\circ} \mathrm{C}$ with high humidity (approximately 90\%) for the first $24 \mathrm{~h}$ in darkness, then transferred back to another moist chamber with a photoperiod of $12 \mathrm{~h}$ under fluorescent lights. Disease severity was assessed and photographed at 7 days after inoculation. For drop inoculation, three 20 - $\mu$ l droplets with different concentrations of conidial suspensions from the indicated strains were placed onto the upper side of the barley seedlings, which were maintained on $4 \%$ (wt/vol) water agar plates. Photographs were taken 5 days after inoculation at $25^{\circ} \mathrm{C}$ (Wang et al. 2013).

\section{RESULTS}

Generation of CHV1-CN280 p29-expressing transformants in wild-type strains of $C$. parasitica and $M$. oryzae. To further explore the function of CHV1-CN280 p29 in C. parasitica and $M$. oryzae, we first attempted to transform the virus-free C. parasitica strain EP155 and wild-type M. oryzae strain Guy11 by a polyethylene glycol-mediated protoplast transformation method, with the expression constructs pCPXHY1::CHV1-CN280-p29 and pCB1532::CHV1-CN280-p29, respectively. Putative transformants containing the above expression vectors were successively transferred on selective medium containing Hyg and sulfonylurea antibiotics, respectively, and stable transformants were obtained. In total, 10 stable transformants for $C$. parasitica and 7 transformants for $M$. oryzae were single-spored and further analyzed by PCR verification assay using genomic DNA and p29 internal primers (p29-F/p29-R). Only two p29-expressing transformants of $C$. parasitica (named A8 and A23) and three of $M$. oryzae (named B7, B9, and B11) showed an approximately 750-bp PCR amplicon consistent with the expected size of the CHV1-CN280 p29 and were taken for further phenotypic characterization. For the RT-PCR assay, the plasmids of pCPXHY1::CHV1-CN280-p29 and pCB1532::CHV1-CN280-p29, as well as cDNA of wild-type EP155 and Guy11, were amplified as positive and negative controls, respectively. C. parasitica $18 \mathrm{~S}$ rRNA and $M$. oryzae actin gene $M G G \_03982$ were used as housekeeping genes. The expected band size of approximately 500 bp was successfully amplified in A8, A23, B7, B9, and B11 transformants (Fig. 1A), which were further validated by qRT-PCR and Southern hybridization. qRT-PCR results showed that the abundance of $p 29$ transcripts increased clearly in the five transformants. The transcription level of CHV1CN280 p29 in wild-type controls EP155 and Guy11 was 0, while the expression levels were $32 \times 102,17 \times 102,16 \times 104,5 \times 104$, and $10 \times 104$ in A8, A23, B7, B9, and B11 transformants, respectively (Fig. 1B). Southern blot experiments with EcoRI, EcoRV, and HindIII-digested DNA showed that the B7 transformant carried three integrations, $\mathrm{B} 11$ together with $\mathrm{A} 8$ had two integrations, and B9 as well as A23 had only one integration (Fig. 1C), suggesting that the CHV1-CN280 p29 has been successfully integrated into the genomes of $C$. parasitica EP155 and M. oryzae Guy11.

CHV1-CN280 p29 expression in $C$. parasitica and $M$. oryzae had no effect on colony growth but different impacts on pigmentation and conidiation. We observed the morphology and tested the colony growth of the CHV1-CN280 p29expressing transformants (A8 and A23) on PDA agar plates. A8 and A23 transformants grew as fast as the wild-type strain EP155 and slower than the hypovirus-containing $C$. parasitica strain CHV1CN280 (Fig. 2B, left). Moreover, A8 and A23 transformants produced fewer orange pigments than the wild-type EP155 strain, with A8 producing fewer orange pigments than A23. As a control, the hypovirulent strain CHV1-CN280 showed an absence of pigmentation (Fig. 2A, left). In addition to reduced orange pigmentation, EP155 cultures transformed with intact CHV1-CN280 p29 (A8 and A23) had strongly suppressed conidiation, with at least 13-fold fewer conidial spores than wild-type strain EP155. The hypovirulent strain CHV1CN280, similar to A8 and A23, exhibited reduced levels of conidiation (Fig. 2C, left). Interestingly, in the qRT-PCR results (Fig. 1B, left), CHV1-CN280 $p 29$ was expressed at a higher level in A8 (>30) than in A23 (>15). This is likely due to A8 having two copies of the CHV1CN280 p29 gene rather than a single copy, as in A23 (Fig. 1C, left). As a result, there is a greater distinction in orange pigmentation and conidiation shown in A8 than A23 (Fig. 2), demonstrating a strong correlation between the expression level of CHV1-CN280 p29 and the degree of phenotypic variance in $C$. parasitica.

Previous studies have reported that CHV1-CN280 has greater potential for biological control of chestnut blight disease because of its mild virulence to $C$. parasitica and high horizontal transmission ability compared with other CHV1 isolates (Deng et al. 2009; Zhang et al. 2012). Preliminary results suggest that CHV1-CN280 p29 expression triggers suppression of orange pigmentation and conidiation in $C$. parasitica, which is a phenomenon known as hypovirulence. It was of considerable interest to determine whether CHV1-CN280 p29 would convert other economically important plant-infecting fungal pathogens to a state of hypovirulence. Thus, we used $M$. oryzae as a representative. No significant differences were observed in the diameter of the fungal colonies grown on the four mediums (CM, PDA, SDC, and V8) after 6 days (Fig. 2B, right), as well as in the colony pigment (Fig. 2A, right). However, CHV1-CN280 p29 expression in M. oryzae led to noticeably enhanced conidia production (Fig. 2C, right), suggesting that CHV1-CN280 p29 plays a different role, either directly or indirectly, in conidiation when expressed in M. oryzae.

Expression of CHV1-CN280 p29 in $C$. parasitica contributed to the reduction of laccase activities. For the CHV1CN280 p29-expressing transformants of C. parasitica with reduced pigmentation and conidiation, we further tested laccase activity, which is well known to be reduced in hypovirulent dsRNA-containing strains. Laccase enzyme activity was examined on PDA plates containing tannic acid and was evaluated by color formation. The hypovirulent strain CHV1-CN280 control showed significantly reduced levels of laccase activity (Fig. 3A). In comparison with the wild-type EP155 strain, the A8 and A23 transformants showed slightly reduced levels of brown coloration and, thus, laccase activity (Fig. 3A). Moreover, three different laccase genes (lac1, lac2, and lac3), which are several developmentally regulated genes affected by the presence of 
dsRNA and responsible for the laccase biosynthesis (Park et al. 2004), were selected to analyze the expression level in the wild type, hypovirulent strain CHV1-CN280, and the A8 and A23 transformants by qRT-PCR. We observed greatly decreased accumulation of lac1, lac2, and lac3 transcripts in the A8 and A23 transformants, while lac1, lac2, and lac3 induction was almost abolished in the hypovirulent strain CHV1-CN280 compared with the wild-type strain EP155 (Fig. 3B). Collectively, these findings indicate that the expression of CHV1-CN280 p29 contributed to the reduction of laccase activity but to a lesser extent than the whole virus in the hypovirulent strains.
Expression of CHV1-CN280 p29 in M. oryzae promoted extracellular laccase activity and contributed to peroxidase activity. The extracellular peroxidase and laccase activities are well known to be correlated with the pathogenicity of M. oryzae. ABTS-inducible laccase expression is measured by the degree of the oxidized dark purple in the reaction. The oxidized dark purple stain around the colonies of Guy11 was less obvious than that of the CHV1-CN280 p29-expressing transformants (B7 and B11) and almost similar to that of B9 (Fig. 4A, indicated by a white circle). Furthermore, the colorimetric assays for filtrates also indicated higher laccase activities in the B7 and B11 transformants (Fig. 4B).

A
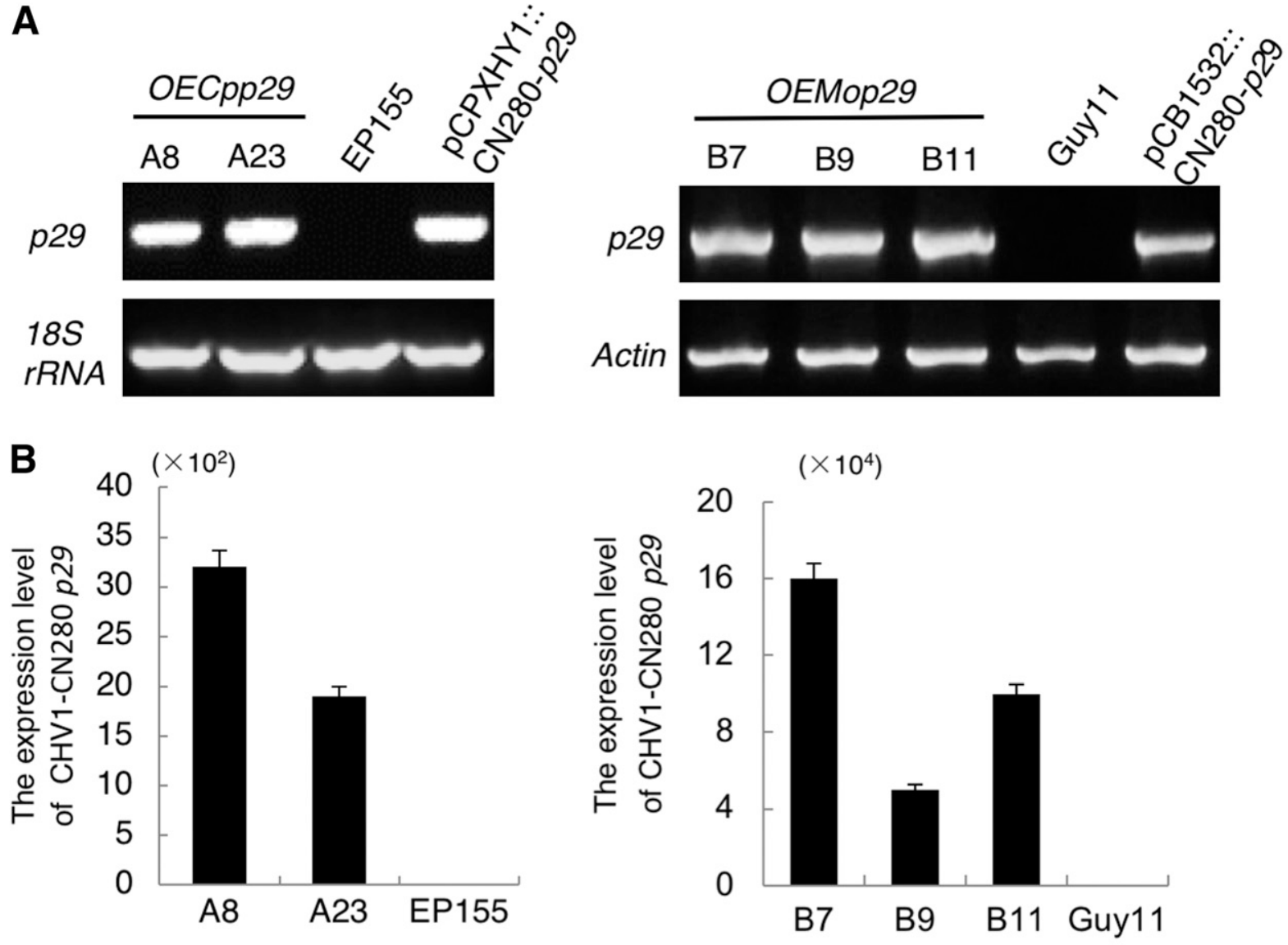

\section{EcoRI}

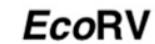

HindIII
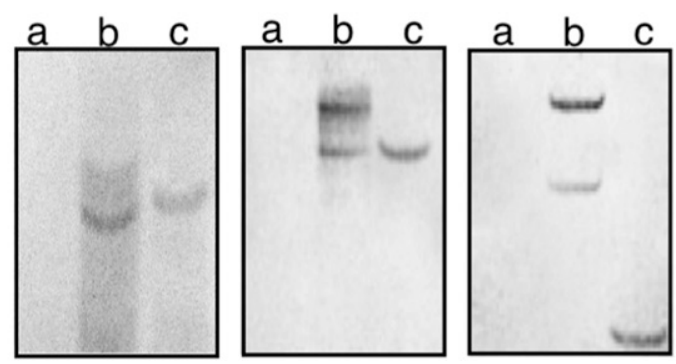

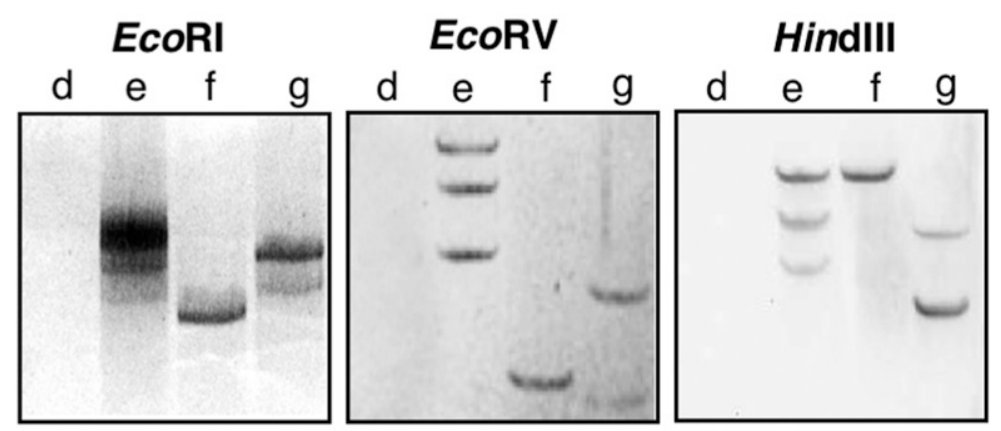

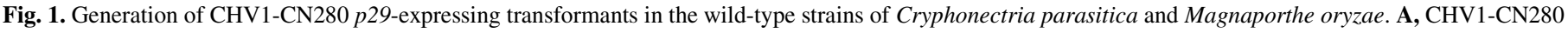

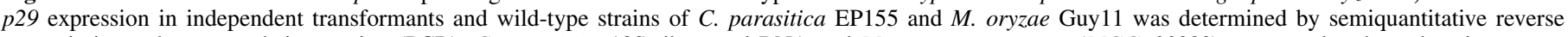

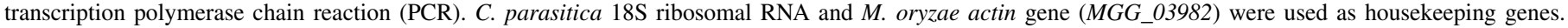

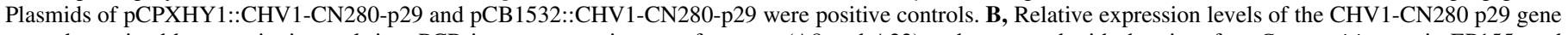

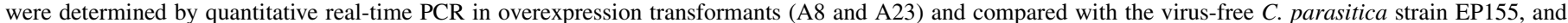

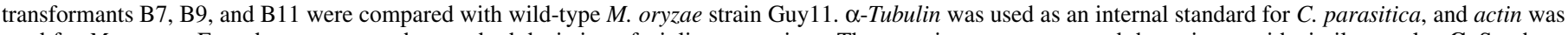

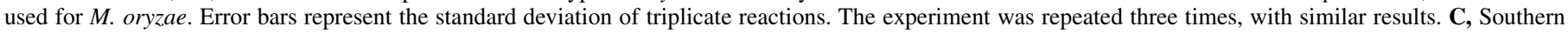

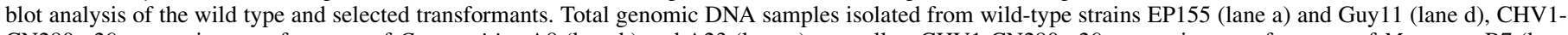

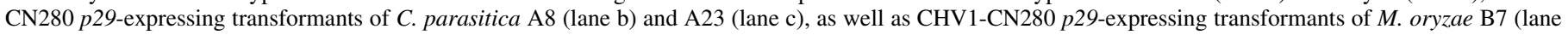

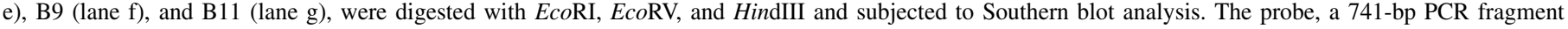
amplified from the cDNA of full-length CHV1-CN280 by primer pair p29-F/p29-R, was exactly the CHV1-CN280 p29 fragment. 
Similarly, qRT-PCR analysis indicated that the transcript levels of laccase-encoding genes ( $M G G \_11608$ and $\left.M G G \_13464\right)$ were significantly increased in B7 and B11 but slightly increased in B9 when compared with the wild-type strain Guy11 (Fig. 4C). Consistent with the expression level of CHV1-CN280 p29 in the three transformants, the laccase activity was greatly increased in B7 and B11 but relatively unchanged in B9 (Figs. 4 and 1B, right). These data suggested that the expression of CHV1-CN280 p29 in $M$. oryzae directly results in increased activity of laccases, in contrast to $C$. parasitica, meaning that CHV1-CN280 p29 may regulate the secretion of laccases in $M$. oryzae.

A second test of extracellular peroxidase activities was conducted using a specific dye named $\mathrm{CR}$, which can oxidize peroxidase from fungal cultures and results in discoloring, thus serving as an indicator for secreted peroxidases (Dunand et al. 2007). The CR degradation halos were observed in all tested strains but were especially apparent in B7 and B9, with a bright golden degradation halo (Fig. 5A). To further test for secreted peroxidases, culture filtrates of the transformants (B7, B9, and B11), along with Guy11, were collected and measured for secretion of extracellular peroxidases by a colorimetric assay. All of the transformants produced a higher colorimetric value at $420 \mathrm{~nm}$ compared with the wild-type strain Guy11 (Fig. 5B). These results suggest CRdegrading activity in the CHV1-CN280 p29-expression transformants of $M$. oryzae. Moreover, the transcript levels of several peroxidase genes (MGG_08200, MGG_07790, MGG_01924, $M G G_{-} 11856$, and $M G G_{-}$13291) were determined by qRT-PCR analysis. Two of five peroxidase genes $\left(M G G_{-} 13291\right.$ and $M G G \_07790$ ) were observed at a significantly higher level in the transformants than in Guy11. A noteworthy increase (>80\%) in $M G G \_13291$ expression level was observed in B7. In contrast, there was an approximately 50\% decrease in the expression levels of $M G G \_01924$ and $M G G \_11856$ in the three transformants compared with Guy11. MGG_08200 was expressed at almost the same level among all tested strains (Fig. 5C). Collectively, these findings indicate that the expression of CHV1-CN280 p29 in M. oryzae had different effects on regulating the transcript levels of extracellular peroxidase-related genes.

CHV1-CN280 p29 expression reduced the virulence of C. parasitica. Hypoviruses cause a variety of interesting and profound phenotypic changes in their fungal hosts. From both a basic and practical perspective, the most interesting change is the attenuation of fungal virulence. Based on the results described above, there is a need to test whether the expression of CN280-p29, in the absence of virus infection, effects hypovirulence. Thus, the CHV1-CN280 p29-expressing transformants (A8 and A23), wildtype strain EP155, and the hypovirulent strain CHV1-CN280 were concurrently inoculated onto standard dormant chestnut tree stems. A8 and A23 both had much larger cankers, with no orange-colored spores, than those produced by the natural hypovirus-infected strain CHV1-CN280 (Fig. 6). Meanwhile, the virulent strain EP155 aggressively produced the largest cankers through the bark surface 5 weeks after inoculation (Fig. 6B). Although the mycelial growth was similar between A8 and A23 transformants and the wild-type strain EP155 on PDA medium (Fig. 2A and B, left), strain EP155 was consistently more aggressive in canker production following inoculation of dormant chestnut stem tissue, producing cankers more than twice the size of those produced by A8 and A23 transformants (Fig. 6). The virulence level of the A8 and A23 transformants was reduced to a lesser extent than that of strain CHV1-CN280 by reintroducing the full-length $p 29$ gene of CHV1CN280 into the virulent strain EP155. Thus, it was discovered that this reduced virulence was due to the expression of CHV1-CN280 p29, suggesting that CHV1-CN280 p29 plays an important role in the hypovirulence of $C$. parasitica.

High-level expression of CHV1-CN280 p29 in M. oryzae may promote pathogenesis. Because CHV1-CN280 p29 expression in $M$. oryzae led to an inverse effect on orange pigmentation, conidiation, and laccase activity compared with results from $C$. parasitica, the conidial suspensions $\left(1 \times 10^{5}\right.$ conidia/ml) of the CHV1-CN280 p29-expressing transformants (B7, B9, and B11) and the wild-type strain Guy11 were sprayed onto 15-day-old susceptible rice seedlings (O. sativa 'CO-39') to perform the pathogenicity assay. At 7 days after inoculation, the CHV1-CN280 p29-expressing transformants (B7, B9, and B11) caused large and extended lesions similar to that of the wild-type Guy11 (Fig. 7A). Disease severity on the rice leaves was evaluated using the "lesion-type" scoring assay ( $\mathrm{Li}$ et al. 2017). Although there were no differences in the production of type 1 or 2 lesions among the three transformants and the wild-type strain Guy11, the production of type 4 or 5 lesions (severe, coalescing) was significantly increased in the B7 and B11 transformants in comparison with wild-type Guy11 (Fig. 7B). B9, which expresses CHV1-CN280 p29 at the lowest level (Fig. 1B), was the only transformant to produce less than or an equal amount of type 4 or 5 lesions and starkly more type 3 lesions than Guy11. These combined results suggested that heterologous expression of CHV1-CN280 p29 in M. oryzae may promote the process of lesion expansion. To further confirm the effect of CHV1-CN280 p29-expressing transformants on virulence, we inoculated three different concentrations of conidial suspension $\left(5 \times 10^{5}, 5 \times 10^{4}\right.$, and $5 \times 10^{3}$ spores $/ \mathrm{ml}$ ) of the indicated strains on detached barley leaves. Consistent with the exacerbated disease symptoms on rice seedlings after inoculation with B7 and B11 transformants, larger lesions developed from B7 transformant inoculations at three different spore concentrations and from B11 transformant inoculation only at the $5 \times 10^{5}$ spore concentration on barley seedlings, compared with wild-type Guy11 (Fig. 7C). Collectively, these results suggested that the high-level expression of CHV1-CN280 p29 in M. oryzae may promote pathogenesis.

\section{DISCUSSION}

In our previous work, we isolated a virulence-attenuating hypovirus, CHV1-CN280, from North China (Deng et al. 2009) and determined its complete genome sequence (Deng et al. 2009; Du et al. 2017). C. parasitica strains infected with the hypovirus CHV1-CN280, while hypovirulent, are more aggressive in colonizing chestnut stem tissues and have higher horizontal transmission abilities between $C$. parasitica isolates belonging to different VC groups as well as higher levels of reduction in orange pigmentation than strains infected with the prototypic hypoviruses CHV1-EP713 or CHV1-Euro7 (Chen and Nuss 1999; Chen et al. 2000; Deng et al. 2009; Zhang et al. 2012). Moreover, similar to CHV1-Euro7-infected strains, CHV1-CN280-infected strains had less of a suppressive effect on mycelial growth, asexual sporulation, and virulence than CHV1-EP713-infected strains (Zhang et al. 2012). In this study, we showed that CHV1-EP713-infected strains were severely reduced in their ability to spread on chestnut tissue, forming small cankers similar to that reported by Chen and Nuss (1999) (Supplementary Fig. S2). Moreover, a preliminary field test showed that, after a 2-year artificial inoculation, CHV1-CN280 successfully colonized in the field and was able to rapidly spread among $C$. parasitica isolates with different vegetative incompatibility types (Zhang et al. 2012). Due to these properties, CHV1$\mathrm{CN} 280$ is a potential candidate for biological control. Here, as an extension of the previous study, we constructed a full-length cDNA clone of CHV1-CN280 into the pMD19-T vector and tried to map the determinants responsible for its high horizontal transmission rate. For CHV1-EP713, a viral protein, p29, was previously reported to enhance vertical hypovirus transmission through asexual spores (Suzuki et al. 2003) and contribute to reduced mycelial growth rates (Suzuki et al. 1999); thus, our study focuses on the functional analysis of $\mathrm{p} 29$.

Protein p29 was considered a viral symptom determinant of many CHV1 strains (Craven et al. 1993; Suzuki et al. 1999, 2003). In this 
A
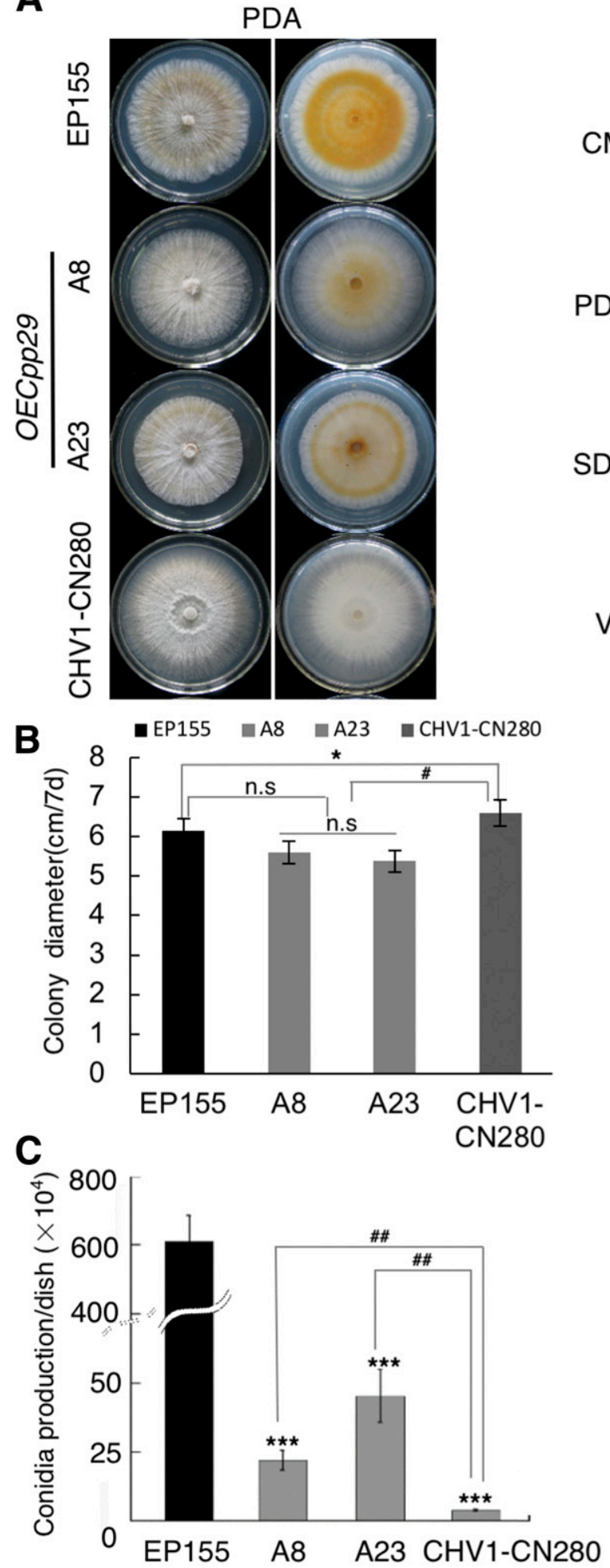
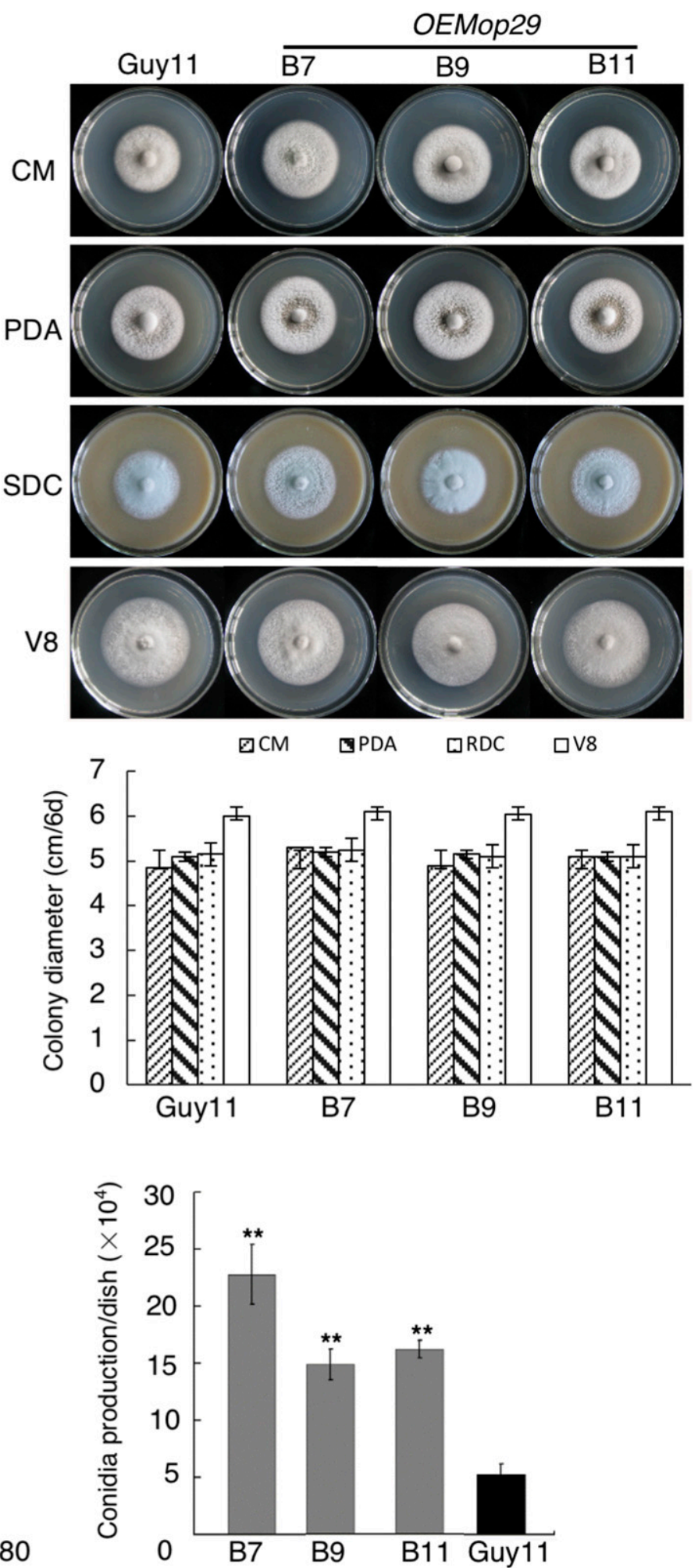

Fig. 2. CHV1-CN280 p29 expression in Cryphonectria parasitica and Magnaporthe oryzae had no effect on colony growth but different impacts on orange pigmentation and conidiation. A, Left panel: colony morphology of the wild-type strain EP155, CHV1-CN280 p29-expressing transformants of C. parasitica (A8 and A23), and hypovirulent strain CHV1-CN280 after 7 days of cultivation on potato dextrose agar (PDA) medium. Right panel: colony morphology of the wildtype strain Guy11 in parallel with CHV1-CN280 p29-expressing transformants of M. oryzae (B7, B9, and B11) after 6 days of cultivation on complete medium (CM), PDA, straw decoction and corn agar (SDC), and V8 medium. This experiment was repeated three times, and representative colonies in the left and right panels were photographed after 7 and 6 days of incubation, respectively. B, Radial growth was measured after incubation for 7 days at $25^{\circ} \mathrm{C}$ in the dark $(C$. parasitica, left) or 6 days at $28^{\circ} \mathrm{C}$ with a 12-h interval photophase (M. oryzae, right). C, Left panel: total number of conidia harvested from $C$. parasitica strains grown on 7.5-cm PDA plates for 14 days. Right panel: total number of conidia harvested from M. oryzae strains grown on 7.5 -cm SDC plates for 7 days in the dark, followed by 3 days of continuous illumination under fluorescent light. Asterisks and number signs denote statistically significant differences among strains tested (*, **, and *** indicate $P<0.05,0.01$, and 0.001 , respectively, versus EP155 or Guy11; \# and \#\# indicate $P<0.01$ and $P<0.001$, respectively, versus CHV1CN280); n.s. = not significant. Data represent three independent experiments, each performed three times. 
article, we characterized CHV1-CN280-encoded p29 using two pathosystems: the natural chestnut stem-C. parasitica interaction and the rice or barley leaf-M. oryzae interaction. The availability of recently developed infectious cDNA clones of L-dsRNA was advantageous in testing the consequences of p29 expression for fungal phenotypes. Our results showed a significant reduction of orange pigmentation, conidiophore formation, laccase activity, and pathogenicity in $p 29$-expressing $C$. parasitica transformants (A8 and A23) compared with the virus-free $C$. parasitica strain EP-155. Importantly, enhanced hypovirulent characteristics were observed in the independent transformant $\mathrm{A} 8$, which exhibited a significantly increased expression level of $p 29$ (Fig. 1B and C). In contrast, A23,

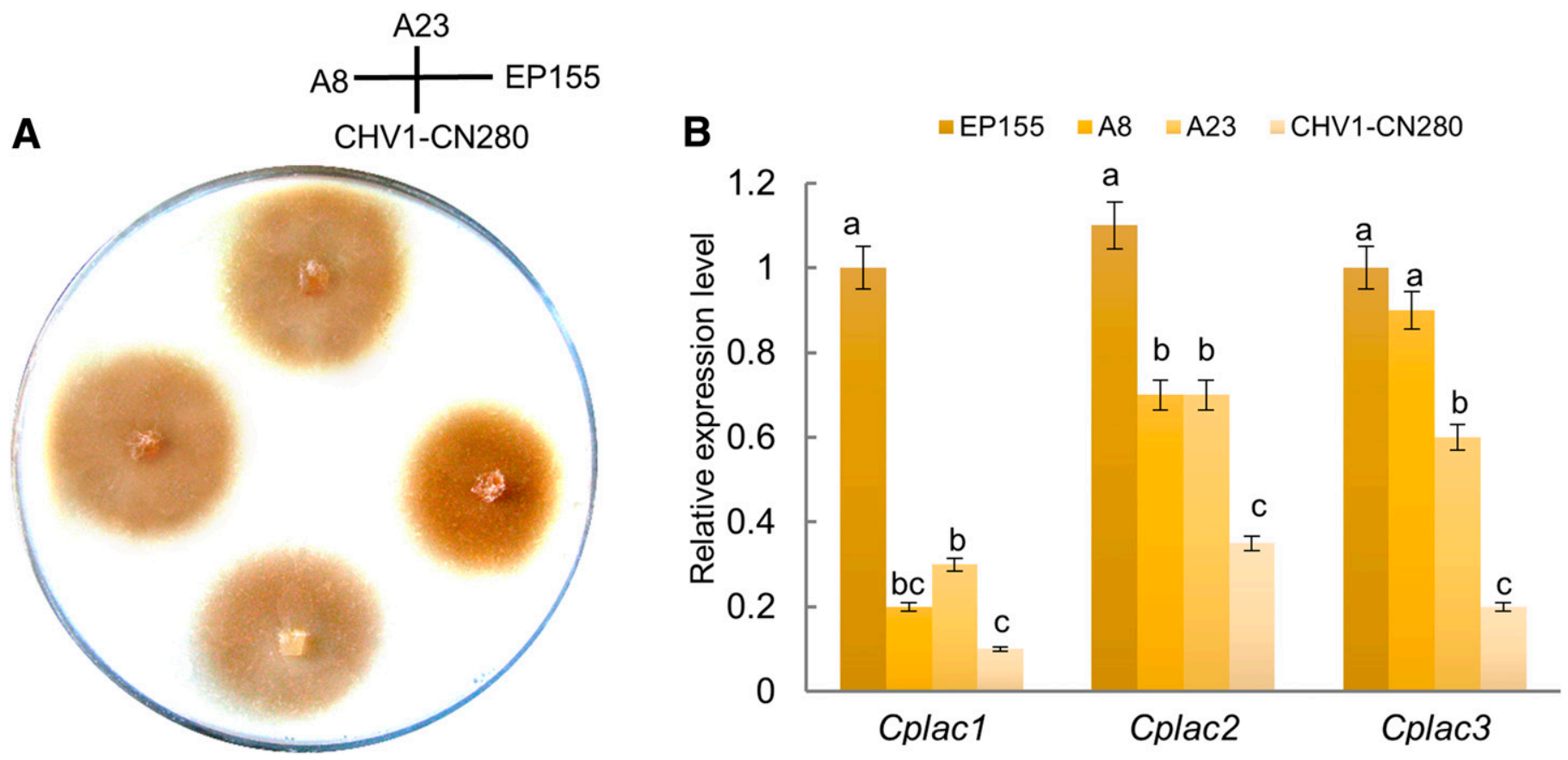

Fig. 3. Expression of CHV1-CN280 p29 in Cryphonectria parasitica contributed to the reduction of laccase activities. A, Laccase activity was monitored on potato dextrose agar medium supplemented with tannic acid as a substrate at $25^{\circ} \mathrm{C}$ for 4 days in the dark. The level of brown coloration correlates with the laccase activity of each strain. B, Expression levels of three different genes (lac1, lac2, and lac3), closely associated with laccase activity in C. parasitica, were determined in the wild type, hypovirulent strain CHV1-CN280, and CHV1-CN280 p29-expressing transformants (A8 and A23) by quantitative real-time polymerase chain reaction. Values are means \pm standard deviations (as error bars) $(n=3)$. Different letters indicate significant differences, as statistically analyzed by Fisher's protected least significant difference test $(P<0.05)$. These experiments were repeated three times with similar results.

A

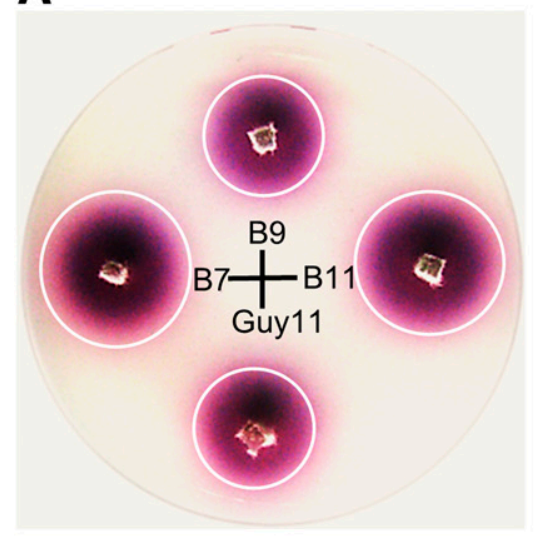

B

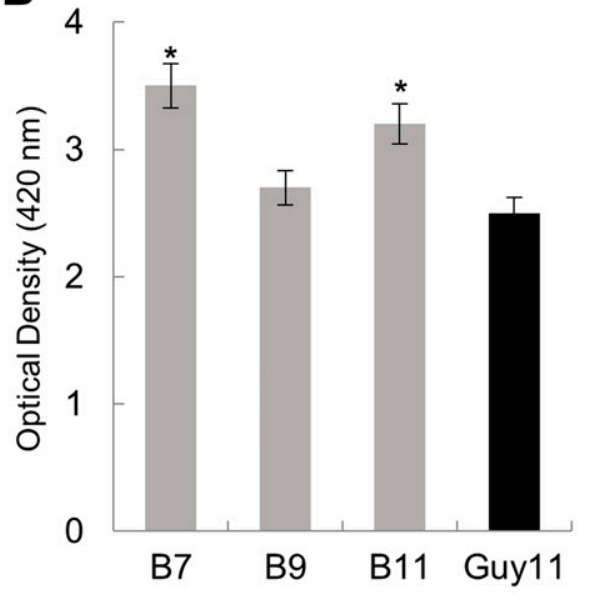

C

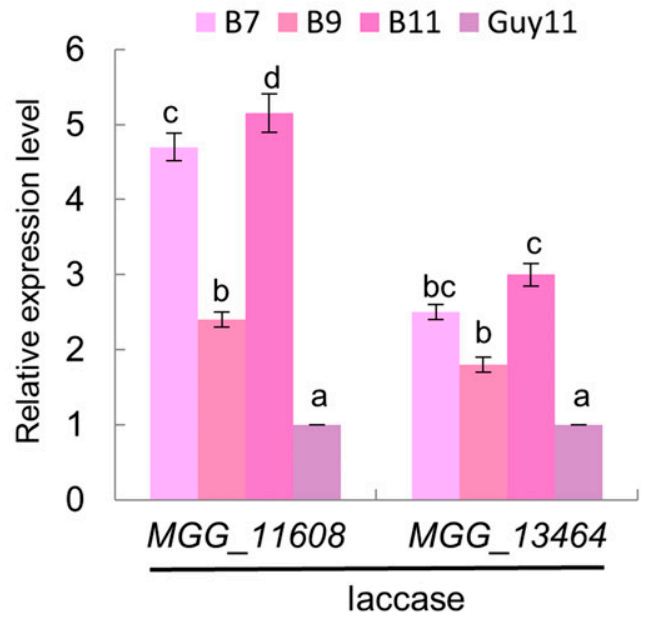

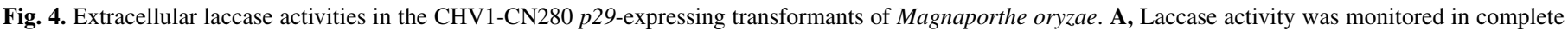

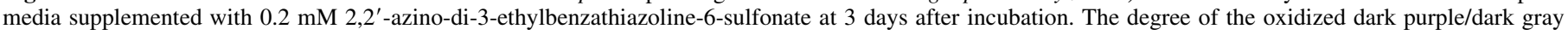

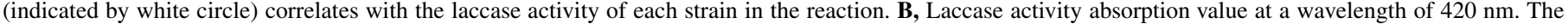

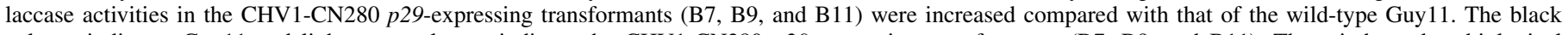

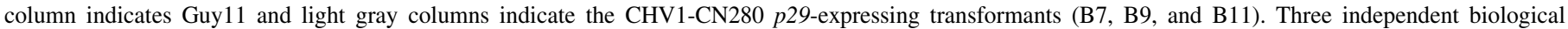

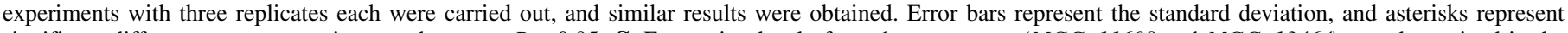

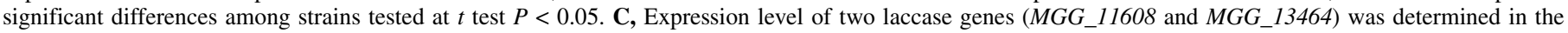

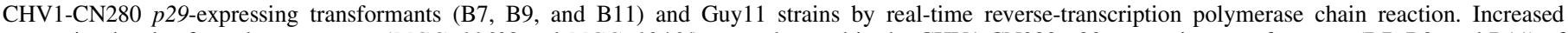

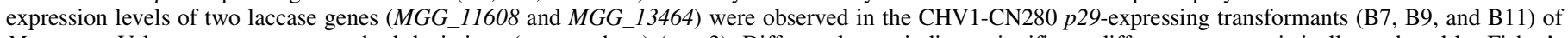

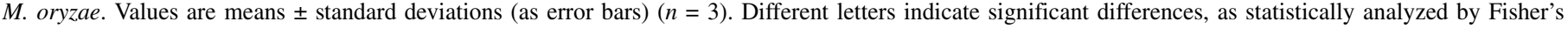
protected least significant difference test $(P<0.05)$. These experiments were repeated three times with similar results. 
due to a lower expression level of $p 29$, showed a lesser extent of the above hypovirulent characteristics. These data suggested a strong correlation between increased expression levels of p29 and enhanced hypovirulence of $C$. parasitica.
Although the p29 sequence of CHV1-CN280 differs starkly when compared with those of the other well-documented CHV1 isolates, both at the nucleotide level and the amino acid level (Supplementary Fig. S1), the phenotypic changes due to CHV1-CN280 p29

\section{A Guy11}

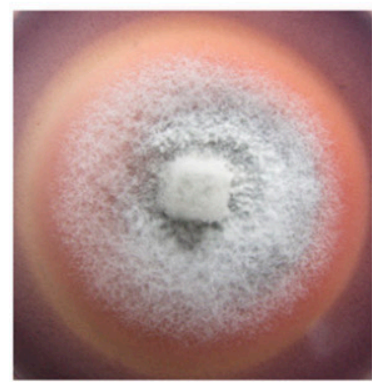

B9
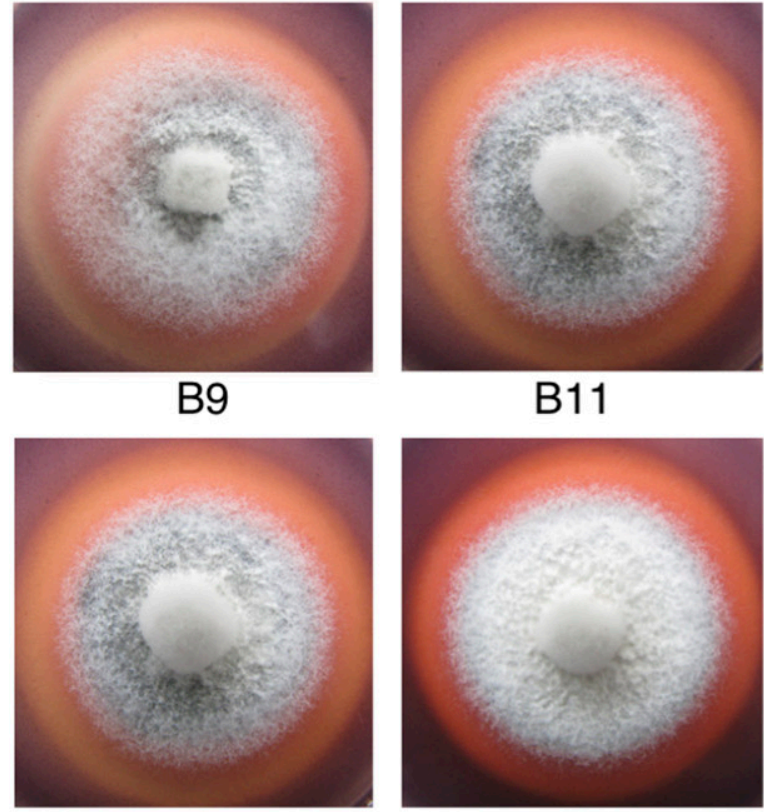

B11

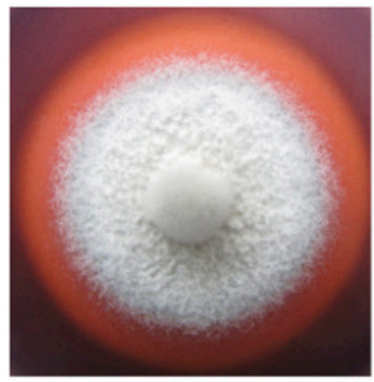

C

음

\section{B7}

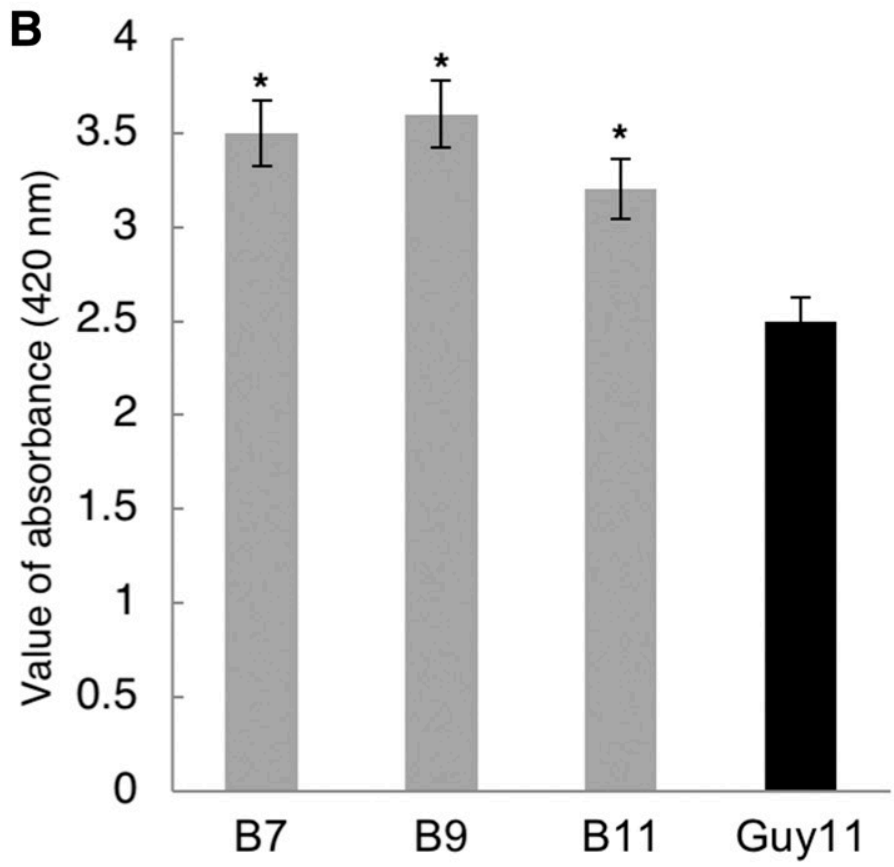

0

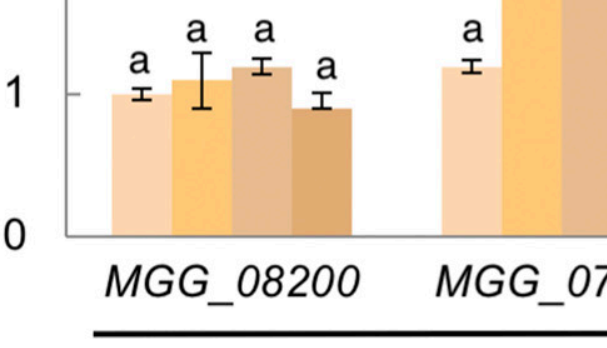

peroxidases with signal peptide

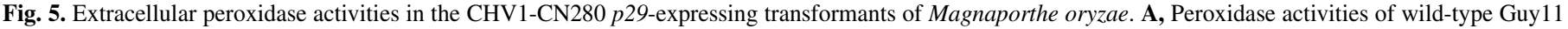

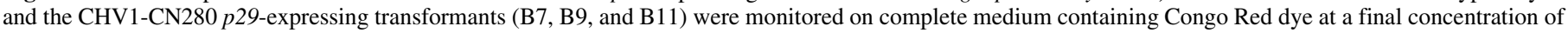

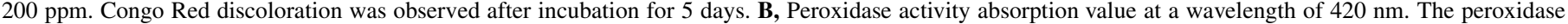

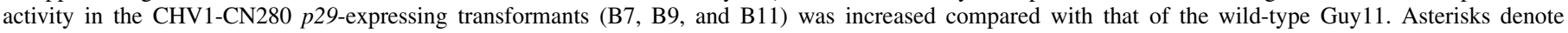

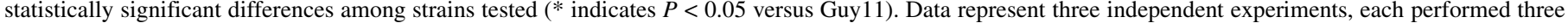

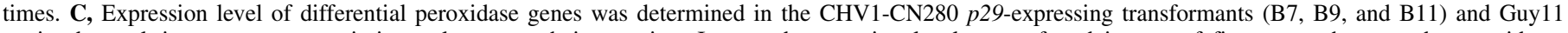

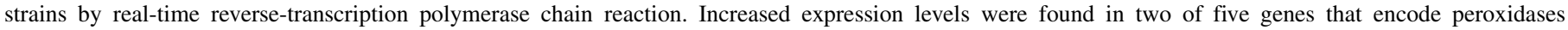

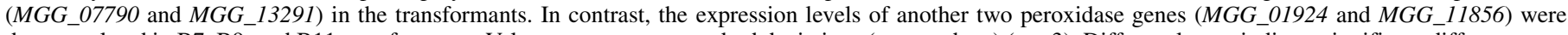

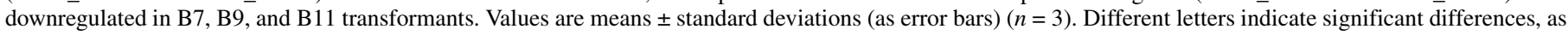

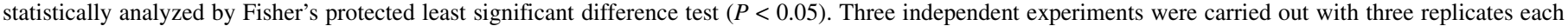
time, and similar results were obtained. Representative results from one of these experiments are shown. 
expression in virus-free $C$. parasitica strain EP-155 were strikingly similar to those previously reported for expression of $p 29$ from other CHV1 isolates. These include $p 29$ expression-mediated suppression of conidiation and pigmentation (Fig. 2), which indicates that the conserved residue of p29 may play the same role as that in other CHV1 isolates. Cys ${ }^{162}$, $\mathrm{His}^{215}, \mathrm{Cys}^{48}$, $\mathrm{Cys}^{70}$, and $\mathrm{Cys}^{72}$ are conserved and have been previously reported to be closely related to the putative protease active site and critical for the function of p29. Our current efforts are focused on generating multiple deletion or substitution mutations of the p29 coding region in the context of the CHV1-CN280 cDNA clone, through loss-of-function and gainof-function assays, to examine whether the conserved residues retain p29's ability to attenuate virulence (hypovirulence) and alter several physiological processes in $C$. parasitica. Of further interest is to determine the potential functional role of the various residues of p29 and the higher horizontal transmission ability observed from CHV1-CN280; for example, the N-terminal portion (1 to 24 amino acids) of p29, which was reported previously to be directly responsible for virus replication (Suzuki et al. 2000) and IRES activities (Chiba et al. 2018), or the $\operatorname{Arg}^{38}$ residue, which is indispensable for the underlying symptoms of p29 (Suzuki et al. 1999).

Finding that CHV1-CN280 p29 was the critical factor conferring hypovirulence when expressed in $C$. parasitica could help to
A
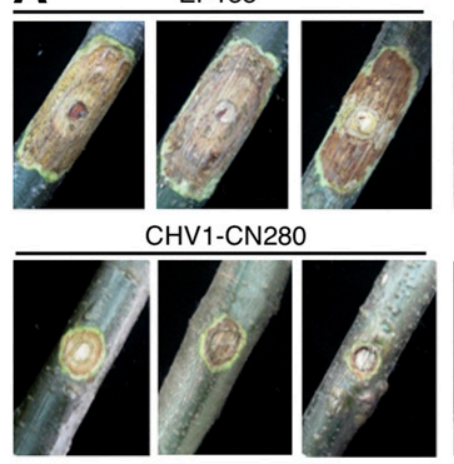

B 14

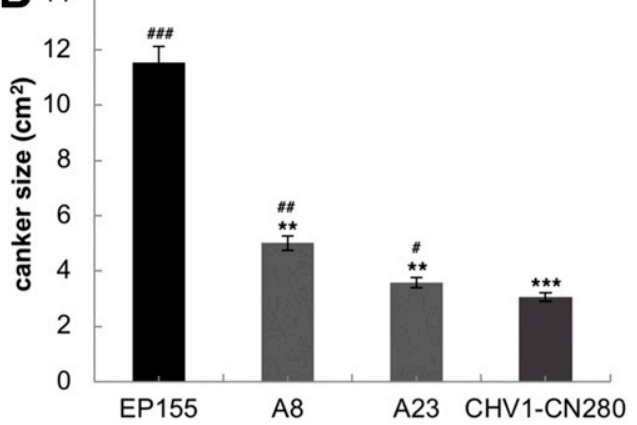

Fig. 6. CHV1-CN280 p29 expression reduced the virulence of Cryphonectria parasitica. A, Virulence assay on dormant Chinese chestnut stems. Mycelial plugs of the wild-type strain EP155, hypovirus CHV1-CN280 strain, and CHV1-CN280 p29-expressing transformants (A8 and A23) were inserted into wounded holes on chestnut stems. Representative cankers were formed at 5 weeks postinoculation. In comparison with wild-type strain EP155, the CHV1CN280 p29-expressing transformants (A8 and A23) formed a much smaller canker with no orange-colored spores and failed to produce the stromal pustules that were formed by EP155 on the bark of inoculated dormant chestnut stems, indicating that the CHV1-CN280 p29-expressing transformants were unable to produce conidia and erupted through the bark of the stems. B, Canker size (in square centimeters) on chestnut plants 5 weeks after inoculation with wild-type strain EP155, hypovirulent strain CHV1-CN280, and CHV1-CN280 p29-expressing transformants (A8 and A23). Measurements of the lesion size (in square centimeters) are means of three replications. Error bars represent the standard deviation. Asterisks and number signs denote 0.01 , respectively, versus EP155; \#\#, \#\#, and \# indicate $P<0.001,0.01$, and 0.05, respectively, versus CHV1-CN280). CHV1-CN280 significant differences among strains tested $(* * *$ and $* *$ indicate $P<0.001$ and potentially overcome the natural barriers to hyphal fusion when using hypovirulent strains as biocontrol tools. Moreover, this prompts us to investigate the possibility of using CHV1-CN280 p29 as a biotechnological application to limit the damage by other fungal pathogens due to the limited strategies available for agricultural diseases. To the best of our knowledge, a number of other studies were performed to study the function of CHV1 ORF Acoded protein p29 in the natural host $C$. parasitica, although no one reported its function in a nonnatural fungal host. $M$. oryzae is a filamentous hemibiotrophic ascomycete that causes rice blast disease, which is one of the most destructive diseases in cultivated rice (Li et al. 2017; Zhang et al. 2011; Zhong et al. 2016). We attempted to engineer hypovirulent $M$. oryzae strains by DNAmediated transformation of $p 29$, the determinant responsible for hypovirulence, as a potential strategy for controlling rice blast disease. Intriguingly, our results showed that the phenotypic changes caused by CHV1-CN280 p29 expression in M. oryzae differed qualitatively from those caused by CHV1-CN280 p29 expression in $C$. parasitica. In contrast, CHV1-CN280 p29 expression in $M$. oryzae resulted in a minor increase in conidiophore formation, laccase activity, and pathogenicity and no changes in vegetative growth or an increase in extracellular peroxidase activities. In addition, one of the three CHV1-CN280
A

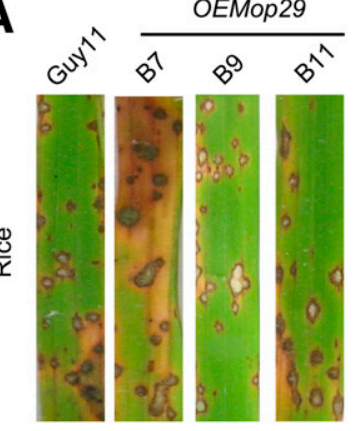

$\mathbf{B}_{14}$

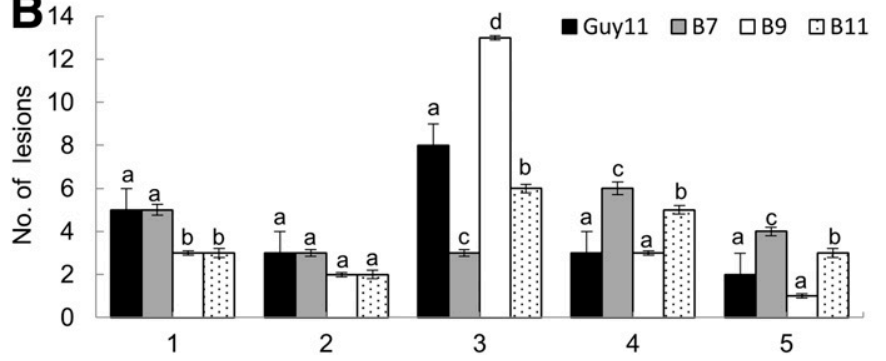

Fig. 7. Pathogenicity assay of Guy11 and the CHV1-CN280 p29-expressing transformants (B7, B9, and B11). A, Rice (Oryza sativa 'CO39') seedlings (left panel) were sprayed with $5 \mathrm{ml}$ of conidial suspensions $\left(1 \times 10^{5}\right.$ conidia/ $\mathrm{ml}$ ) from the wild-type Guy11 and the CHV1-CN280 p29-expressing transformants (B7, B9, and B11). Lesions were photographed and scored at 7 days postinoculation (dpi). Experiments were repeated twice with similar results. In total, 30 healthy rice seedlings were inoculated with conidial suspensions of each indicated strain. B, Quantitative analysis of disease severity caused by the wild-type Guy11 and the CHV1-CN280 p29-expressing transformants (B7, B9, and B11). Disease symptoms on rice seedlings exhibited various disease severity $(1=$ pinhead-sized brown specks, $2=1.5-\mathrm{mm}$ brown spots, $3=2$ - to 3-mm gray spots with brown margins, $4=$ many elliptical gray spots longer than $3 \mathrm{~mm}$, and $5=$ coalesced lesions infecting $50 \%$ or more of the leaf area). Three independent biological experiments yielded similar results. Values are means \pm standard deviations (as error bars) $(n=3)$. Different letters indicate significant differences, as statistically analyzed by Fisher's protected least significant difference test $(P<0.05)$. C, Detached barley (Hordeum vulgare 'Four-arris') seedlings (right panel) were drop inoculated with different concentrations of conidial suspensions from the indicated strains. Photographs were taken at $5 \mathrm{dpi}$, and experiments were repeated twice with similar results. In total, 30 barley leaves were inoculated with conidial suspensions of each indicated strain. The B7 transformant showed stronger virulence compared with the wild-type strain (Guy11) and two other transformants (B9 and B11), as manifested by larger lesions at $5 \mathrm{dpi}$. 
p29-expressing transformants in M. oryzae, B7, which had the highest expression level of CN280-p29, exhibited remarkably increased conidiophore formation, laccase activity, and pathogenicity, suggesting that there appears to be a linkage between increased expression levels of $p 29$ and enhanced morphological and physiological changes.

Transgenic expression of CHV1-CN280 p29 in M. oryzae showed different trends of changes in some phenotypic traits from those in $C$. parasitica. In this regard, we speculate that CHV1CN280 p29 may disturb a unique regulatory pathway in C. parasitica rather than a basic regulatory pathway conserved in a diverse range of fungi. However, Segers et al. (2006) have previously reported that CHV1-EP713 p29 could act as a suppressor of RNA silencing, which has been shown to serve as a conserved defense mechanism in fungi (Qiao et al. 2013; Xiong et al. 2014). There was a significant difference in the amino acid sequences of p29 between CHV1-CN280 and the three other CHV1 isolates. It seems quite possible that CHV1-CN280 p29 may not act as a suppressor of RNA silencing due to only $61.7 \%$ amino acid sequence similarity between CHV1-CN280 p29 and CHV1-EP713 p29. Different activities have already been observed to have effects on host virulence; namely, expression of CHV1-CN280 p29 resulted in hypovirulence, whereas no effect on virulence was found for CHV1-EP713 p29 (Craven et al. 1993) (Fig. 6). In this regard, this will offer support for the hypothesis proposed above. In the future, it will be of considerable interest to test the impact of p29 in other fungi in addition to M. oryzae and determine, in detail, whether CHV1-CN280 p29 functions as a suppressor of RNA silencing. Previous studies showed that some host proteins could act as "helpers" to facilitate effector functions during pathogenesis, whereas others are "targets" (Kong et al. 2015; Win et al. 2012). Host proteins also manipulate the activity of pathogen effectors. For example, a soybean cyclophilin GmCYP1 is reported to be a host helper recruited by a Phytophthora effector Avr3b to become an active virulence protein in plant cells. The nudix hydrolase activity of Avr3b, which is essential for virulence function, was only activated after Avr3b was delivered into soybean cells by the soybean cyclophilin (Kong et al. 2015). Coaker et al. (2005) also showed that Arabidopsis cyclophilin ROC1 is required to activate the cysteine protease activity of the bacterial effector AvrRpt2. Thus, p29-mediated modulation of fungal phenotypes may also be dependent on the specific interaction between p29 and a special host helper, which only exists in $C$. parasitica rather than $M$. oryzae. Further investigations will be conducted to identify fungal host components that associate with p29 in $C$. parasitica by performing yeast two-hybrid screens and coimmunoprecipitation assays.

In summary, we have demonstrated that CHV1-CN280 p29 plays an essential role in hypovirus-induced symptoms, including reducing levels of orange pigmentation, suppressing sporulation, and reducing laccase activities and virulence in $C$. parasitica, while having some opposite effects in $M$. oryzae. To our knowledge this is the first report characterizing the functions of CHV1-CN280 p29 in $C$. parasitica and a nonnatural host, $M$. oryzae, by heterologous expression. These findings will significantly facilitate future studies on the p29 mechanism of action.

\section{ACKNOWLEDGMENTS}

We thank Q. Zhuge at Nanjing Forestry University for kindly sharing lab equipment and J. Waletich and K. Tao at the Oregon State University for their editorial assistance.

\section{LITERATURE CITED}

Anagnostakis, S. L. 1982. Biological control of chestnut blight. Science 215: 466-471.

Castillo, A., Cottet, L., Castro, M., and Sepúlveda, F. 2011. Rapid isolation of mycoviral double-stranded RNA from Botrytis cinerea and Saccharomyces cerevisiae. Virol. J. 8:38.
Chen, B., Chen, C. H., Bowman, B. H., and Nuss, D. L. 1996. Phenotypic changes associated with wild-type and mutant hypovirus RNA transfection of plant pathogenic fungi phylogenetically related to Cryphonectria parasitica. Phytopathology 86:301-310.

Chen, B., Geletka, L. M., and Nuss, D. L. 2000. Using chimeric hypoviruses to fine-tune the interaction between a pathogenic fungus and its plant host. J. Virol. 74:7562-7567.

Chen, B., and Nuss, D. L. 1999. Infectious cDNA clone of hypovirus CHV1Euro7: A comparative virology approach to investigate virus-mediated hypovirulence of the chestnut blight fungus Cryphonectria parasitica. J. Virol. 73:985-992.

Chiba, S., Jamal, A., and Suzuki, N. 2018. First evidence for internal ribosomal entry sites in diverse fungal virus genomes. MBio 9:e02350-17.

Churchill, A., Ciuffetti, L., Hansen, D., Van Etten, H., and Van Alfen, N. 1990. Transformation of the fungal pathogen Cryphonectria parasitica with a variety of heterologous plasmids. Curr. Genet. 17:25-31.

Coaker, G., Falick, A., and Staskawicz, B. 2005. Activation of a phytopathogenic bacterial effector protein by a eukaryotic cyclophilin. Science 308 : 548-550.

Craven, M. G., Pawlyk, D. M., Choi, G. H., and Nuss, D. L. 1993. Papain-like protease p29 as a symptom determinant encoded by a hypovirulenceassociated virus of the chestnut blight fungus. J. Virol. 67:6513-6521.

Dean, R., Van Kan, J. A., Pretorius, Z. A., Hammond-Kosack, K. E., Di Pietro, A., Spanu, P. D., Rudd, J. J., Dickman, M., Kahmann, R., Ellis, J., and Foster, G. D. 2012. The Top 10 fungal pathogens in molecular plant pathology. Mol. Plant Pathol. 13:414-430.

Deng, Q., Ye, Y., Miao, M., Fang, Q., Li, T., and Wang, K. 2009. The horizontal transmission of Cryphonectria hypovirus 1 (CHV1) is affected by virus strains. Chin. Sci. Bull. 54:3053-3060.

Du, Y., Lin, Y., Zhou, X., Wang, K., Fang, S., and Deng, Q. 2017. Full-length sequence and genome analysis of CHV1-CN280, a North China isolate of Cryphonectria hypovirus 1. Arch. Virol. 162:1811-1818.

Dunand, C., Crèvecoeur, M., and Penel, C. 2007. Distribution of superoxide and hydrogen peroxide in Arabidopsis root and their influence on root development: Possible interaction with peroxidases. New Phytol. 174: 332-341.

Eusebio-Cope, A., Sun, L., Tanaka, T., Chiba, S., Kasahara, S., and Suzuki, N. 2015. The chestnut blight fungus for studies on virus/host and virus/virus interactions: From a natural to a model host. Virology 477:164-175.

Fisher, M. C., Hawkins, N. J., Sanglard, D., and Gurr, S. J. 2018. Worldwide emergence of resistance to antifungal drugs challenges human health and food security. Science 360:739-742.

Gao, K., Xiong, Q., Xu, J., Wang, K., and Wang, K. 2013. CpBirl is required for conidiation, virulence and anti-apoptotic effects and influences hypovirus transmission in Cryphonectria parasitica. Fungal Genet. Biol. 51: 60-71.

Kim, D., Rigling, D., Zhang, L., and Van Alfen, N. 1995. A new extracellular laccase of Cryphonectria parasitica is revealed by deletion of Lac1. Mol. Plant-Microbe Interact. 8:259-266.

Kong, G., Zhao, Y., Jing, M., Huang, J., Yang, J., Xia, Y., Kong, L., Ye, W., Xiong, Q., Qiao, Y., Dong, S., Ma, W., and Wang, Y. 2015. The activation of Phytophthora effector Avr3b by plant cyclophilin is required for the Nudix hydrolase activity of Avr3b. PLoS Pathog. 11:e1005139.

Li, L., Chen, X., Zhang, S., Yang, J., Chen, D., Liu, M., Zhang, H., Zheng, X., Wang, P., and Peng, Y. 2017. MoCAP proteins regulated by MoArk1mediated phosphorylation coordinate endocytosis and actin dynamics to govern development and virulence of Magnaporthe oryzae. PLoS Genet. 13:e1006814.

Lin, H., Lan, X., Liao, H., Parsley, T. B., Nuss, D. L., and Chen, B. 2007. Genome sequence, full-length infectious cDNA clone, and mapping of viral double-stranded RNA accumulation determinant of hypovirus CHV1EP721. J. Virol. 81:1813-1820.

MacDonald, W., and Fulbright, D. 1991. Biological control of chestnut blight: Use and limitations of transmissible hypovirulence. Plant Dis. 75:656-661.

Mir, A. A., Park, S. Y., Sadat, M. A., Kim, S., Choi, J., Jeon, J., and Lee, Y. H. 2015. Systematic characterization of the peroxidase gene family provides new insights into fungal pathogenicity in Magnaporthe oryzae. Sci. Rep. 5: Article 11831

Nuss, D. L. 2005. Hypovirulence: Mycoviruses at the fungal-plant interface. Nat. Rev. Microbiol. 3:632-642.

Park, S. M., Choi, E. S., Kim, M. J., Cha, B. J., Yang, M. S., and Kim, D. H. 2004. Characterization of HOG1 homologue, CpMK1, from Cryphonectria parasitica and evidence for hypovirus-mediated perturbation of its phosphorylation in response to hypertonic stress. Mol. Microbiol. 51:1267-1277.

Puhalla, J. E., and Anagnostakis, S. L. 1971. Genetics and nutritional requirements of Endothia parasitica. Phytopathology 61:169-173.

Qi, Z., Wang, Q., Dou, X., Wang, W., Zhao, Q., Lv, R., Zhang, H., Zheng, X., Wang, P., and Zhang, Z. 2012. MoSwi6, an APSES family transcription factor, interacts with MoMps1 and is required for hyphal and conidial 
morphogenesis, appressorial function and pathogenicity of Magnaporthe oryzae. Mol. Plant Pathol. 13:677-689.

Qiao, Y., Liu, L., Xiong, Q., Flores, C., Wong, J., Shi, J., Wang, X., Liu, X., Xiang, Q., and Jiang, S. 2013. Oomycete pathogens encode RNA silencing suppressors. Nat. Genet. 45:330-333.

Rigling, D., Heiniger, U., and Hohl, H. R. 1989. Reduction of laccase activity in dsRNA-containing hypovirulent strains of Cryphonectria (Endothia) parasitica. Phytopathology 79:219-223.

Rimbaud, L., Papaix, J., Rey, J. F., Barrett, L. G., and Thrall, P. H. 2018. Assessing the durability and efficiency of landscape-based strategies to deploy plant resistance to pathogens. PLoS Comput. Biol. 14:e1006067.

Sasaki, A., Onoue, M., Kanematsu, S., Suzaki, K., Miyanishi, M., Suzuki, N., Nuss, D. L., and Yoshida, K. 2002. Extending chestnut blight hypovirus host range within Diaporthales by biolistic delivery of viral cDNA. Mol. Plant-Microbe Interact. 15:780-789.

Segers, G. C., van Wezel, R., Zhang, X., Hong, Y., and Nuss, D. L. 2006. Hypovirus papain-like protease p29 suppresses RNA silencing in the natural fungal host and in a heterologous plant system. Eukaryot. Cell 5:896-904.

Shapira, R., Choi, G. H., and Nuss, D. L. 1991. Virus-like genetic organization and expression strategy for a double-stranded RNA genetic element associated with biological control of chestnut blight. EMBO J. 10:731-739.

Suzuki, N., Chen, B., and Nuss, D. L. 1999. Mapping of a hypovirus p29 protease symptom determinant domain with sequence similarity to potyvirus HC-Pro protease. J. Virol. 73:9478-9484.

Suzuki, N., Geletka, L. M., and Nuss, D. L. 2000. Essential and dispensable virus-encoded replication elements revealed by efforts to develop hypoviruses as gene expression vectors. J. Virol. 74:7568-7577.

Suzuki, N., Ghabrial, S. A., Kim, K. H., Pearson, M., Marzano, S. Y. L., Yaegashi, H., Xie, J., Guo, L., Kondo, H., Koloniuk, I., and Hillman, B. I., and ICTV Report Consortium. 2018. ICTV Virus Taxonomy Profile: Hypoviridae. J. Gen. Virol. 99:615-616.

Suzuki, N., Maruyama, K., Moriyama, M., and Nuss, D. L. 2003. Hypovirus papain-like protease p29 functions in trans to enhance viral double-stranded RNA accumulation and vertical transmission. J. Virol. 77:11697-11707.

Talbot, N. J., Ebbole, D. J., and Hamer, J. E. 1993. Identification and characterization of $M P G 1$, a gene involved in pathogenicity from the rice blast fungus Magnaporthe grisea. Plant Cell 5:1575-1590.
Urayama, S., Fukuhara, T., Moriyama, H., Tohe, A., and Kawamoto, S. 2014. Heterologous expression of a gene of Magnaporthe oryzae chrysovirus 1 strain A disrupts growth of the human pathogenic fungus Cryptococcus neoformans. Microbiol. Immunol. 58:294-302.

Urayama, S., Ohta, T., Onozuka, N., Sakoda, H., Fukuhara, T., Arie, T., Teraoka, T., and Moriyama, H. 2012. Characterization of Magnaporthe oryzae chrysovirus 1 structural proteins and their expression in Saccharomyces cerevisiae. J. Virol. 86:8287-8295.

Wang, J., Du, Y., Zhang, H., Zhou, C., Qi, Z., Zheng, X., Wang, P., and Zhang, Z. 2013. The actin-regulating kinase homologue MoArk1 plays a pleiotropic function in Magnaporthe oryzae. Mol. Plant Pathol. 14:470-482.

Win, J., Chaparrogarcia, A., Belhaj, K., Saunders, D. G., Yoshida, K., Dong, S., Schornack, S., Zipfel, C., Robatzek, S., and Hogenhout, S. A. 2012. Effector biology of plant-associated organisms: Concepts and perspectives. Cold Spring Harb. Symp. Quant. Biol. 77:235-247.

Xie, J., Havens, W. M., Lin, Y. H., Suzuki, N., and Ghabrial, S. A. 2016. The victorivirus Helminthosporium victoriae virus $190 \mathrm{~S}$ is the primary cause of disease/hypovirulence in its natural host and a heterologous host. Virus Res. 213:238-245.

Xie, J., and Jiang, D. 2014. New insights into mycoviruses and exploration for the biological control of crop fungal diseases. Annu. Rev. Phytopathol. 52:45-68.

Xiong, Q., Ye, W., Choi, D., Wong, J., Qiao, Y., Tao, K., Wang, Y., and Ma, W. 2014. Phytophthora suppressor of RNA silencing 2 is a conserved RxLR effector that promotes infection in soybean and Arabidopsis thaliana. Mol. Plant-Microbe Interact. 27:1379-1389.

Zhang, H., Tang, W., Liu, K., Huang, Q., Zhang, X., Yan, X., Chen, Y., Wang, J., Qi, Z., Wang, Z., Zheng, X., Wang, P., and Zhang, Z. 2011. Eight RGS and RGS-like proteins orchestrate growth, differentiation, and pathogenicity of Magnaporthe oryzae. PLoS Pathog. 7:e1002450.

Zhang, L., Gao, K., Deng, Q., Ye, Y., and Wang, K. 2012. Biological control potential of the hypovirus CHV1-CN280 against chestnut blight. Chin. J. Biol. Control 28:80-86.

Zhong, K., Li, X., Le, X., Kong, X., Zhang, H., Zheng, X., Wang, P., and Zhang, Z. 2016. MoDnm1 dynamin mediating peroxisomal and mitochondrial fission in complex with MoFis1 and MoMdv1 is important for development of functional appressorium in Magnaporthe oryzae. PLoS Pathog. 12:e1005823. 